\title{
Identificação De Assimetrias No Rotor De Máquinas De Indução Com Baixa Carga Usando O Envelope Da Corrente Do Estator Com Taxa De Amostragem Reduzida
}

\author{
Josemar de Souza Moreira*. Isabela Oliveira Zaparoli*. João Pedro Carvalho Moreira*. Ana Carolina Santos \\ Araújo*. Paulo Cezar Monteiro Lamim Filho*. \\ * LAMET - Laboratório de Máquinas Elétricas e Transformadores \\ Departamento de Engenharia Elétrica \\ UFSJ - Universidade Federal de São João del-Rei \\ Pça. Frei Orlando, 170 - Centro - 36307-352 - São João del-Rei, MG, Brasil \\ E-mails: js_moreira99@yahoo.com.br,oliveirazaparoli@gmail.com,mcjpedro@gmail.com, \\ anacarolinasantosaraujo@hotmail.com,plamim@yahoo.com
}

\begin{abstract}
Diagnosing the broken bars in induction motors operating at low loads is a hard task reported by authors in the literature. This paper presents a method of reducing the sampling frequency for the processing of current signals, looking for a minimum sufficient sampling required to diagnose a failure of broken bars. Two technical of signals demodulation, the Hilbert Transform and the method of empirical demodulation, were used for the invitation of envelopes that carry the asymmetry information in the motor. The method was used to detect a single broken bar. The results show how the decimation technique significantly reduces the number of operations, data storage space and time required to calculate the Fast Fourier Transform without loss of information. This approach provides a better performance of embedded systems for diagnostics based on the amplitude modulation characteristic.

Resumo: Diagnosticar barras quebradas em motores de indução operando com baixas cargas é uma dificuldade relatada por diversos autores na literatura. Este artigo apresenta um estudo da redução da frequência de amostragem para o processamento de sinais de corrente, buscando identificar a mínima frequência de amostragem suficiente para diagnosticar a falha de barras quebradas. Duas técnicas de demodulação de sinais, a Transformada de Hilbert e um método de demodulação empírica, foram usadas para obtenção dos envelopes que carregam as informações de assimetria no motor. $\mathrm{O}$ método foi utilizado para detecção de uma única barra quebrada. Os resultados mostram como a técnica de decimação reduz significativamente o número de operações, espaço de armazenamento de dados e o tempo necessário para o cálculo da Transformada Rápida de Fourier sem perda de informação. Esta abordagem pode proporcionar um melhor desempenho de sistemas embarcados para o diagnóstico baseadas na característica de modulação em amplitude.
\end{abstract}

Keywords: Decimation; Signal processing; Frequency spectrum; Broken bars; Induction motors.

Palavras-chaves: Decimação; Espectro de corrente; Envelopes; Barras quebradas; Motores de indução.

\section{INTRODUÇÃO}

As máquinas elétricas assíncronas de rotor gaiola são amplamente utilizadas para o acionamento de cargas mecânicas uma vez que podem trabalhar em ambientes agressivos, pois não possuem anéis no rotor e tem baixo custo de aquisição e de manutenção em comparação às demais máquinas elétricas. Assim, os motores de indução do tipo gaiola de esquilo representam aproximadamente $85 \%$ dos motores instalados nas indústrias (Abd-el-Malek, 2017). Devido a suas aplicações, os motores estão sujeitos a estresses térmicos, mecânicos e elétricos que podem ocasionar em falhas durante sua operação. Nos últimos 30 anos, pesquisadores e empresas têm desenvolvido várias técnicas que podem ser usadas para detecção de falhas em motores elétricos. O objetivo principal é ter uma técnica confiável, mas também de fácil aplicação industrial, de modo a não colocar o operador em contato direto com a máquina monitorada. Um método bastante utilizado é a Análise de Padrões da Corrente do Motor (MCSA), (Puche-Panadero, 2012).

A técnica MCSA, baseia-se na análise espectral da corrente do estator, utilizando como ferramenta matemática a Transformada Rápida de Fourier (FFT) que possibilita a identificação de padrões que correspondem a assimetrias ou falhas no rotor de motores de indução.

Porém, a MCSA tem suas limitações. Trabalhos como Akin (2011), Gyftakis (2014) e Gao (2015) relatam a dificuldade de diagnosticar a falha de barras quebradas quando o motor opera com baixas cargas, isto ocorre devido a assinatura de falha ser de baixa frequência. A mesma dificuldade é relatada por Puche-Panadero (2009), Sapena-Bano (2015), que 
utilizam uma frequência de amostragem de $5.12 \mathrm{kHz}$ e tempo de amostragem de 100 segundos para conseguir uma resolução em frequência de $0,001 \mathrm{~Hz}$. Isso implica no aumento do espaço em memória e maior tempo de processamento dos sinais, exigindo grande esforço computacional.

A identificação de falha de barras quebradas com a análise espectral pode ser melhorada com técnicas de demodulação de sinais como apresentado por Puche-Panadero (2009) e Aydin (2011) que utilizaram a transformada de Hilbert combinada a FFT para identificar a frequência de falha sobre o espectro do sinal demodulado. Outros autores propõem o uso de ferramentas de demodulação de sinais como o algoritmo Fault Frequency Highlighting (FFH), desenvolvido por Lamim Filho (2016) que proporciona um realce das frequências características de falha, tornando-as mais claramente identificáveis, e a demodulação empírica proposta por Batista (2016) com o algoritmo ED.

Quando o objetivo é fazer a análise espectral dos envelopes de corrente, torna-se desnecessária a aquisição dos sinais com elevada frequência de amostragem como relatado na literatura (Puche-Panadero 2009, Sapena-Bano 2015, Gyftakis 2016). Isso porque a falha de barras quebradas produz sobre os sinais de corrente, modulações em amplitude de baixa frequência.

Nesse sentido, para contornar o problema de espaço em memória e tempo de processamento, o número de amostras de um sinal pode ser reduzido por um processo de decimação, como em Chakkor (2015), que utiliza a decimação em um algoritmo para detecção de falhas de turbinas eólicas. Os resultados do estudo demonstraram o desempenho do algoritmo permitindo a identificação de harmônicos de alta resolução com tempo mínimo de computação e menor custo de memória.

A proposta desta pesquisa consiste em reduzir a frequência de amostragem dos sinais de corrente, a fim de obter a mínima frequência de amostragem suficiente para a detecção da falha de barras quebradas pela análise espectral dos envelopes. São utilizadas duas técnicas de demodulação diferentes, a clássica transformada de Hilbert e o algoritmo ED. Com o processo de decimação, espera-se diminuir ao máximo possível a taxa de amostragem dos sinais de corrente, sem comprometer a informação do sinal de falha, visando reduzir o esforço computacional e o espaço ocupado em memória.

\section{FUNDAMENTAÇÃO TEÓRICA}

\subsection{Falha de Barras Quebradas elou Trincadas do Motor de Indução}

A ocorrência de barras quebradas em motores de indução provoca modulações em amplitude nos sinais de corrente do estator. Estas modulações possuem frequências características de falha que podem ser obtidas através de um processo de demodulação de sinais (Bonnett, 2008).

Tais frequências características de falha, são representadas por (1), na qual as constantes $s$ e $f$ referem-se, respectivamente, ao escorregamento e a frequência de alimentação, sendo $k$ um número inteiro positivo.

$$
f_{b r}=(1 \pm 2 s k) f
$$

\subsection{Transformada de Hilbert}

A Transformada de Hilbert (HT) é uma técnica de análise de sinais bem conhecida e amplamente usada em diferentes campos científicos, como transmissão de sinais, processamento de dados geofísicos, e demodulação de sinais em motores de indução (Puche-Panadero, 2009).

A transformada de Hilbert de um sinal modulado qualquer representado por $x(t)$, é definida por (2).

$H[x(t)]=\frac{1}{\pi} \int_{-\infty}^{+\infty} \frac{x(t)}{t-\tau} d \tau$

Pode-se criar um sinal analítico $z(t)$ com parte real e imaginária, sendo a parte real o próprio sinal e a parte imaginária a Transformada de Hilbert deste sinal. Na forma polar o sinal analítico é definido por (3).

$$
z(t)=A(t) e^{j \theta(t)}
$$

Sendo que $A(t)$ e $\theta(t)$ representam a amplitude e a fase instantânea do sinal analítico, respectivamente. $O$ envelope ou sequência de tempo modulada de $z(t)$ é definido como o módulo do sinal analítico.

\subsection{Algoritmo ED}

As informações contidas no envelope dos sinais de corrente podem ser obtidas por um processo de demodulação de sinais que permite separar as frequências que carregam as informações de falha. Este processo pode ser realizado através da aplicação de diversas ferramentas de processamento de sinais. A demodulação empírica apresentada em Batista (2016), baseia-se na obtenção dos extremos locais de uma sequência de tempo modulada e posterior interpolação de máximos e mínimos para a construção do envelope. Os passos para a formulação do algoritmo ED são mostrados a seguir:

Passo 1- Remover o nível CC de x.

Passo 2- Construir a sequência incompleta $\hat{\mathrm{x}}_{\mathrm{ED}}$ e $\mathrm{t}_{\mathrm{ED}}$ como:

$\hat{\mathrm{x}}_{\mathrm{ED}}=\{\}$ e $\mathrm{t}_{\mathrm{ED}}=\{\}$

para $\mathrm{n}=1,2, \ldots, \mathrm{N}-2$

se $x[n+1] \geq x[n]$ e $x[n+1] \geq x[n+2]$ e

$\mathrm{x}[\mathrm{n}+1] \geq 0$

então $x[n+1]$ é um máximo local,

$\hat{\mathrm{x}}_{\mathrm{ED}}=\left\{\hat{\mathrm{x}}_{\mathrm{ED}} \mathrm{x}[\mathrm{n}+1]\right\}$,

$e t_{E D}=\left\{t_{E D} t[n+1]\right\}$

se não se $x[n+1] \leq x[n]$ e $x[n+1] \leq x[n+2]$ e

$\mathrm{x}[\mathrm{n}+1] \leq 0$ 
então $x[n+1]$ é um mínimo local, $\widehat{\mathrm{x}}_{\mathrm{ED}}=$

$\left\{\hat{\mathrm{x}}_{\mathrm{ED}}-\mathrm{x}[\mathrm{n}+1]\right\}, \mathrm{e} \mathrm{t}_{\mathrm{ED}}=\left\{\mathrm{t}_{\mathrm{ED}} \mathrm{t}[\mathrm{n}+1]\right\}$

Atualize as sequências $\hat{\mathrm{X}}_{\mathrm{ED}}$ e $\mathrm{t}_{\mathrm{ED}}$ :

$\hat{\mathbf{x}}_{\mathrm{ED}}=\left\{\hat{\mathrm{X}}_{\mathrm{ED}}[1] \hat{\mathrm{X}}_{\mathrm{ED}}[2] \hat{\mathbf{x}}_{\mathrm{ED}} \hat{\mathrm{X}}_{\mathrm{ED}}[\right.$ end -1$\left.] \hat{\mathrm{X}}_{\mathrm{ED}}[\mathrm{end}]\right\}$

$\mathbf{t}_{\mathrm{ED}}=\left\{\left(\mathrm{t}[1]-\left(\mathrm{t}_{\mathrm{ED}}[2]-\mathrm{t}[1]\right)\right)\left(\mathrm{t}[1]-\left(\mathrm{t}_{\mathrm{ED}}[1]-\mathrm{t}[1]\right)\right) \mathbf{t}_{\mathrm{ED}} \ldots\right.$

$\ldots\left(\mathrm{t}[\right.$ end $]+\left(\mathrm{t}[\right.$ end $]-\mathrm{t}_{\mathrm{ED}}[$ end $\left.\left.]\right)\right)$

$\left(\mathrm{t}[\mathrm{end}]+\left(\mathrm{t}[\mathrm{end}]-\mathrm{t}_{\mathrm{ED}}[\right.\right.$ end -1$\left.\left.\left.]\right)\right)\right\}$

\subsection{Decimação}

A técnica de decimação consiste em reduzir o número de amostras originais de um sinal por um fator inteiro $p$. Tal processo pode ser feito tomando uma amostra e eliminando as demais amostras $p-1$, ou preferencialmente calculando a média da amostra $p$, pois, desta forma reduz-se o ruído de quantificação. Nesse sentido, a decimação funciona como um filtro passa baixa, eliminando a banda de alta frequência do sinal original. Ao longo da frequência de amostragem reduzida e largura de banda, a sequência de dados também é diminuída em $p$ vezes (De Jesus, 2017).

O exemplo a seguir mostra a aplicação da técnica em um dado sinal no domínio do tempo discretizado a uma frequência de amostragem de $2500 \mathrm{~Hz}$, como ilustrado na Fig. 1. Ao aplicar um fator de decimação $p_{i}=4$ obtêm-se o gráfico da Fig. 2.

Nota-se que o sinal possui mesmo formato, porém um número menor de pontos, como se estivesse sido amostrado a uma frequência de $625 \mathrm{~Hz}$. Desta forma, com um número menor de pontos é possível representar o mesmo sinal mantendo suas informações de magnitude e forma de onda.

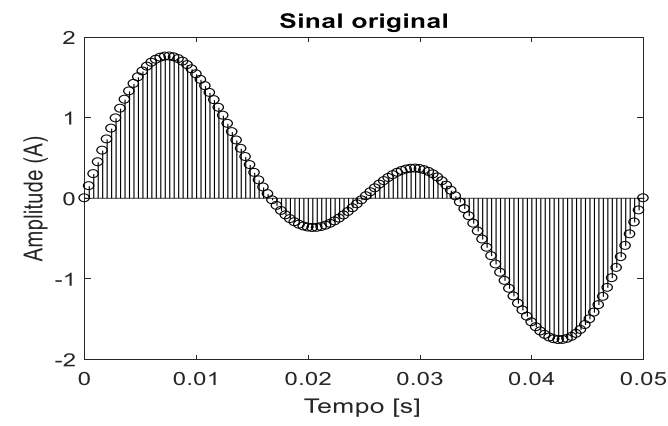

Fig. 1 Sinal amostrado a $2500 \mathrm{~Hz}$.

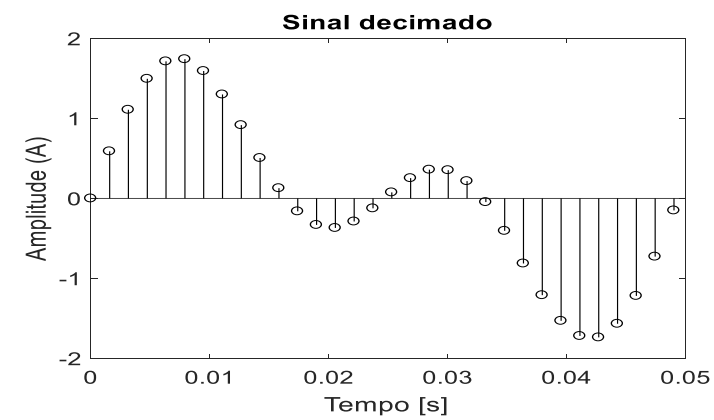

Fig. 2 Sinal decimado por um fator 4.
Na Figura 3, tem-se o fluxograma do algoritmo utilizado para a detecção da falha. A partir de um sinal de corrente coletado em uma das fases do estator, é aplicado um filtro passa faixa (40-70 Hz). Em seguida o sinal é decimado no tempo por um fator $p_{i}=2^{N}$, sendo $\mathrm{N}$ um número inteiro. Dessa forma, ao variar $\mathrm{N}$ o número de amostras dos sinais será sempre um múltiplo de potência de 2 .

Após a aplicação da decimação, são obtidos os envelopes do sinal com a transformada de Hilbert e o algoritmo ED. Posteriormente aplica-se a FFT obtendo o espectro do envelope.

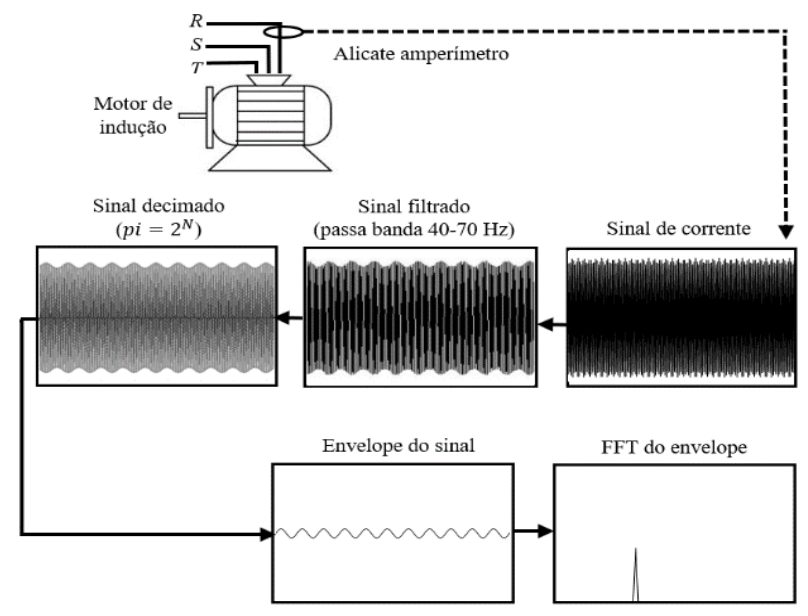

Fig. 3 Fluxograma do algoritmo.

Para realização das etapas descritas foi utilizado o software Matlab. A aquisição dos sinais de corrente de estator do motor de indução foi realizada em uma bancada (Figura 4) composta por um motor de indução trifásico WEG $3 \mathrm{CV}, 220$ $\mathrm{V}, 60 \mathrm{~Hz}, 4$ polos e velocidade nominal de $1735 \mathrm{rpm}$. O motor em questão foi acoplado a um gerador CC ligado a um banco de resistores que em conjunto, funcionam como um sistema de carga para o motor de indução. Entre o motor de indução e o gerador foi inserido um transdutor de torque, ligado a um torquímetro digital para medir o nível de torque e garantir uma aquisição de dados padronizada.

Os sinais de corrente foram coletados por meio de um alicate amperímetro (A622 AC/DC - $100 \mathrm{~Hz}$ Tectronix), ligado a uma placa de aquisição (NI PCI- 4461 da National Instruments. A placa possui duas entradas analógicas simultâneas de 24-bits (taxa de amostragem máxima de 80 $\mathrm{kHz}$ ) e duas saídas analógicas simultâneas de 24-bits (taxa de amostragem de $204,8 \mathrm{kS} / \mathrm{s}$ ) com faixa de entrada de $\pm 316 \mathrm{mV}$ até $42,4 \mathrm{~V}$. Além disso, ela possui filtro anti-aliasing até 92 $\mathrm{kHz}$. 


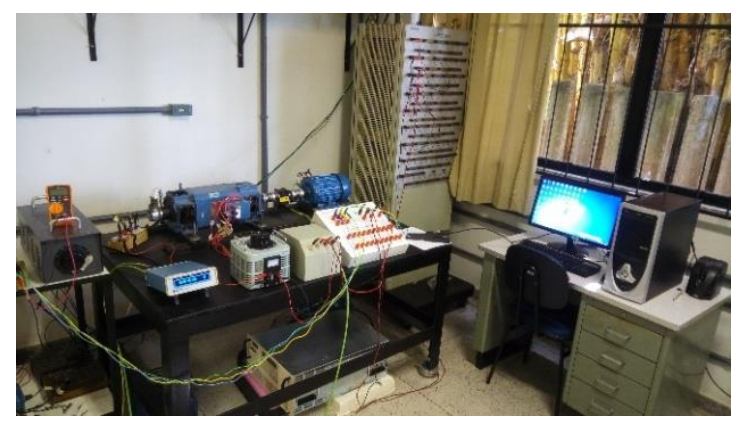

Fig. 4 Bancada de testes experimentais.

Os sinais de corrente foram coletados a uma frequência de amostragem de $5,12 \mathrm{kHz}$, durante 102,4 segundos, gerando um total de 524288 amostras, para a condição de uma barra do rotor quebrada como mostra a Figura 5.

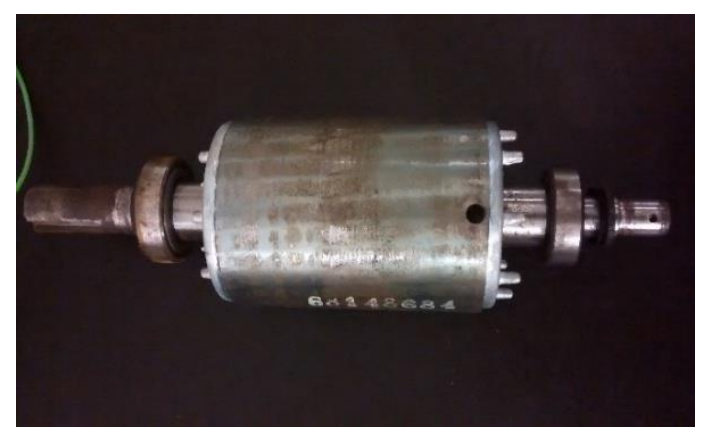

Fig. 5 Rotor com uma barra quebrada.

\section{RESULTADOS}

Foram analisados os sinais de corrente para o motor operando a $20 \%, 10 \%$ e $0 \%$ de carga. Na Tabela 1 tem-se o número de amostras e as respectivas frequências de amostragem dos sinais para cada fator de decimação aplicado.

O método de demodulação empírica ED e a transformada de Hilbert apresentaram desempenho semelhante para a frequência de amostragem reduzida até $640 \mathrm{~Hz}$. A partir deste valor, somente a transformada de Hilbert foi capaz de manter as características espectrais do envelope, permitindo que a frequência de falha fosse facilmente identificada para um sinal com frequência de amostragem de $160 \mathrm{~Hz}$.

Tabela 1. Número de amostras e frequências dos sinais decimados.

\begin{tabular}{|c|c|c|}
\hline $\begin{array}{c}\text { Fator de } \\
\text { decimação }\end{array}$ & $\begin{array}{c}\text { Número de } \\
\text { amostras }\end{array}$ & Frequência de amostragem $(\mathrm{Hz})$ \\
\hline 1 & 524288 & 5120 \\
\hline 2 & 262144 & 2560 \\
\hline 4 & 131072 & 1280 \\
\hline 8 & 65536 & 640 \\
\hline 16 & 32768 & 320 \\
\hline 32 & 16384 & 160 \\
\hline
\end{tabular}

\subsection{Resultados da Transformada de Hilbert}

Na Fig. 6, tem-se os espectros dos envelopes obtidos pela transformada de Hilbert para o motor operando a $20 \%, 10 \%$ e $0 \%$ de carga respectivamente, obtidos com o sinal original coletado a 5,12 kHz. Na Fig. 7 tem-se os espectros dos envelopes, obtidos com o sinal decimado por um fator $p i=8$, resultando em uma frequência de amostragem de $640 \mathrm{~Hz}$. Na Fig. 8 tem-se os espectros dos envelopes, obtidos com o sinal decimado por um fator $p i=32$, resultando em uma frequência de amostragem de $160 \mathrm{~Hz}$.

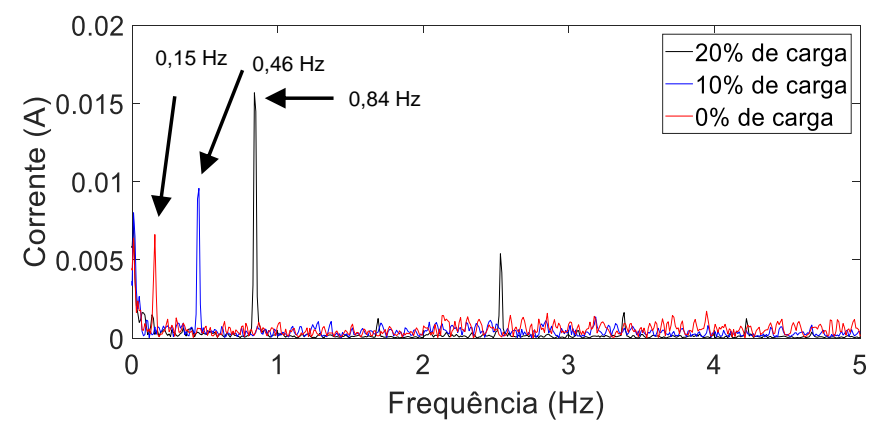

Fig. 6 Espectro do envelope HT para $f s=5120 \mathrm{~Hz}$ e o motor operando a $20 \%, 10 \%$ e $0 \%$ de carga.

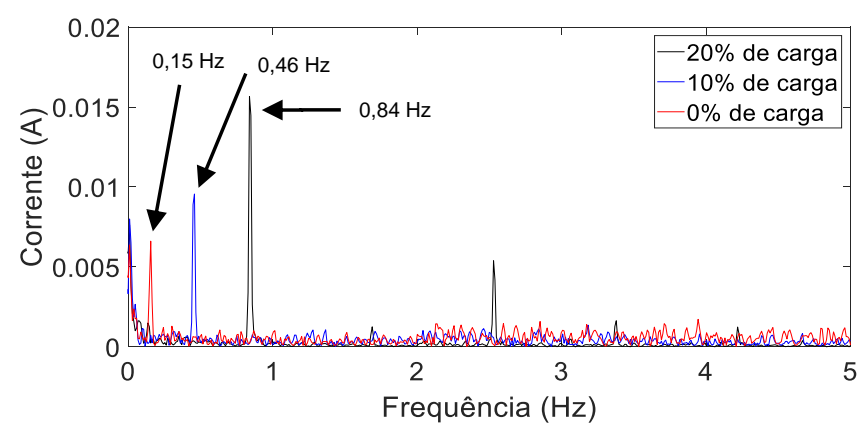

Fig. 7 Espectro do envelope HT para $f_{s}=640 \mathrm{~Hz}$ e o motor operando a $20 \%, 10 \%$ e $0 \%$ de carga.

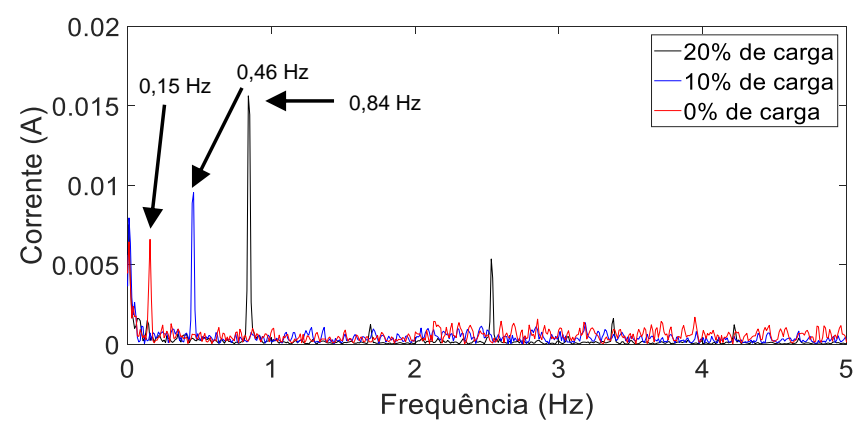

Fig. 8 Espectro do envelope HT para $f s=160 \mathrm{~Hz}$ e o motor operando a $20 \%, 10 \%$ e $0 \%$ de carga.

\subsection{Resultados do Algoritmo ED}

Na Fig. 9, tem-se os espectros dos envelopes para o motor operando a $20 \%, 10 \%$ e $0 \%$ de carga respectivamente, obtidos com o sinal original coletado a $5,12 \mathrm{kHz}$. Na Fig. 10 tem-se os espectros dos envelopes, obtidos com o sinal decimado por um fator $p i=8$, resultando em uma frequência de amostragem de $640 \mathrm{~Hz}$. Quando é aplicado a um fator $p i=$ 16, o algoritmo ED já não é capaz de obter as modulações reais do sinal de falha, principalmente para a condição do motor operando a vazio. Nessas condições o espectro do envelope é comprometido como mostra a Fig. 11.

Ambas as técnicas de demodulação de sinais quando aliadas a FFT, mostram-se ótimas ferramentas para detecção da falha 
de barras quebradas em motores operando com baixas cargas e até mesmo a vazio. A maneira de obter os envelopes dos sinais de corrente se difere bastante entre as duas técnicas. Enquanto a transformada de Hilbert é mais sensível à presença de múltiplas modulações, enquanto que o algoritmo ED extrai essencialmente as modulações sobre a frequência fundamental, referente a falha de barras quebradas como mostram as Figs. 12 e 13.

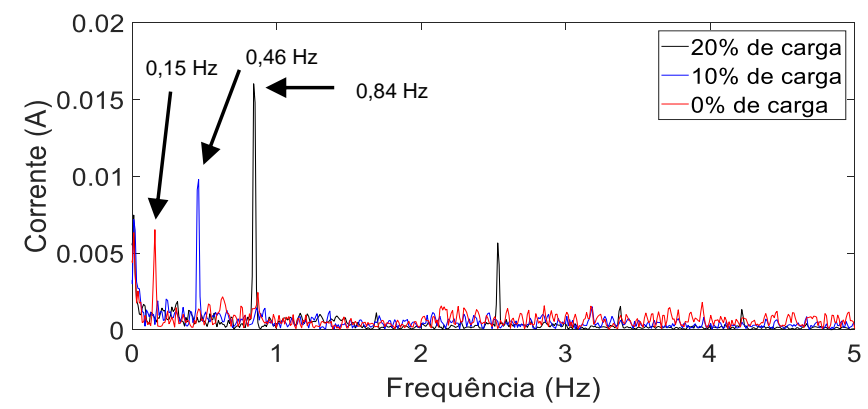

Figura 9 - Espectro do envelope ED para $f s=5120 \mathrm{~Hz}$ e o motor operando a $20 \%, 10 \%$ e $0 \%$ de carga.

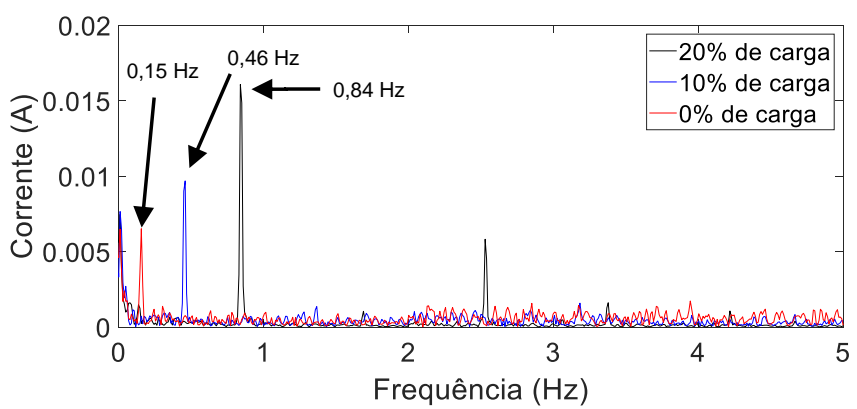

Fig. 10 Espectro do envelope ED para $f s=640 \mathrm{~Hz}$ e o motor operando a $20 \%, 10 \%$ e $0 \%$ de carga.

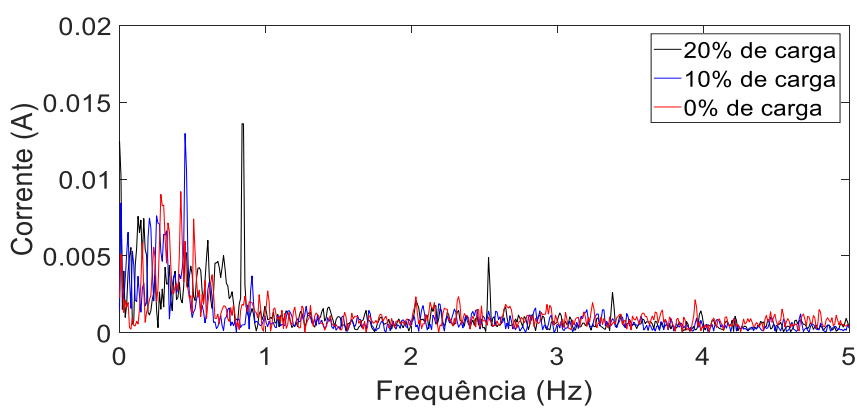

Fig. 11 Espectro do envelope ED para $f_{s}=160 \mathrm{~Hz}$ e o motor operando a $20 \%, 10 \%$ e $0 \%$ de carga.

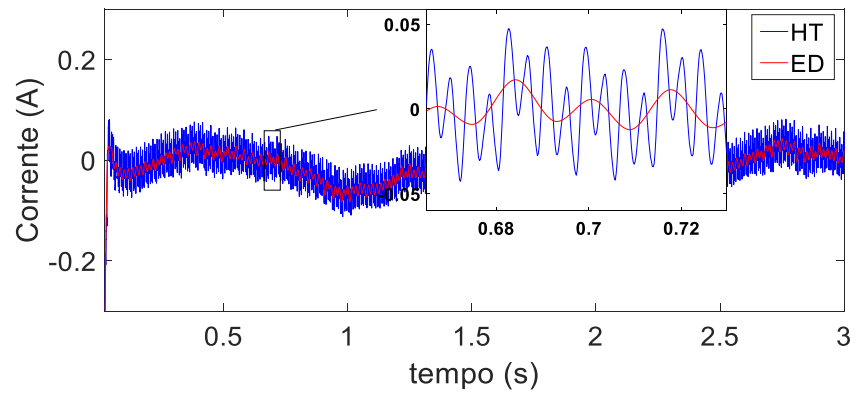

Fig. 12 Envelopes de um sinal de corrente: em azul com a transformada de Hilbert e em vermelho com o algoritmo ED.

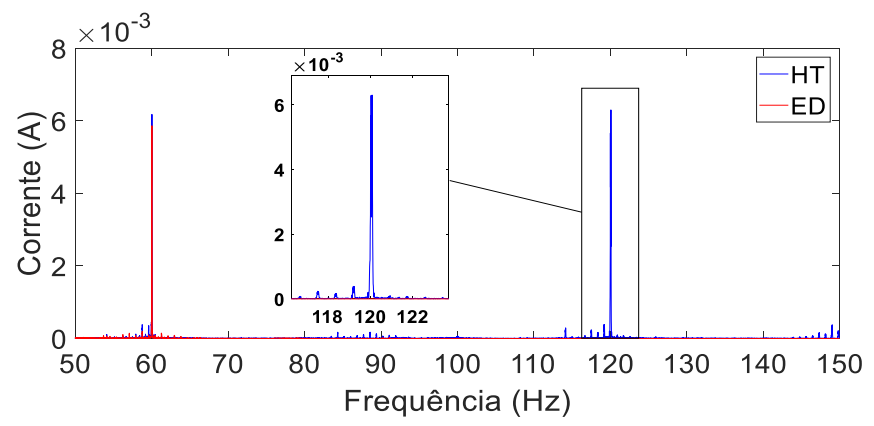

Fig. 13 Espectro dos envelopes da transforma Hilbert e do algoritmo ED.

\subsection{Esforço Computacional}

A redução da frequência de amostragem, proporciona um ganho em termos de esforço computacional. Com o sinal representado por um número menor de amostras, o número de operações do algoritmo como um todo é drasticamente reduzido. Para exemplificar, tomou-se para análise a etapa de cálculo da FFT que, conforme Lathi (2006) realiza $\operatorname{Nlog}_{2} \mathrm{~N}$ adições complexas e (N/2) $\log _{2} N$ multiplicações complexas para o cálculo de um vetor $N$.

A Tabela 2 relaciona o número de operações necessárias para o cálculo da FFT de um envelope, para a cada fator de decimação aplicado. O máximo fator de decimação aplicável ao algoritmo ED reduz em 9 vezes o número de operações necessárias para o cálculo da FFT, enquanto que esta redução é da ordem de 60 vezes com a transformada de Hilbert.

Tabela 2. Número de operações necessárias para o cálculo da FFT.

\begin{tabular}{|c|c|c|c|}
\hline $\begin{array}{c}\text { Fator de } \\
\text { decimação }\end{array}$ & $\begin{array}{c}\text { Número de } \\
\text { multiplicações }\end{array}$ & $\begin{array}{c}\text { Número } \\
\text { de } \\
\text { adições }\end{array}$ & $\begin{array}{c}\text { Total de } \\
\text { operações }\end{array}$ \\
\hline 1 & 4980736 & 9961472 & 14942208 \\
\hline 2 & 2359296 & 4718592 & 7077888 \\
\hline 4 & 1114112 & 2228224 & 3342336 \\
\hline 8 & 524288 & 1048576 & 1572864 \\
\hline 16 & 245760 & 491520 & 737280 \\
\hline 32 & 79496 & 158990 & 238490 \\
\hline
\end{tabular}

\section{CONCLUSÃO}

Ao reduzir sucessivas vezes a frequência de amostragem, percebeu-se que é possível diagnosticar a presença de barras quebradas de um motor com um número significativamente menor de amostras de um sinal, sem perder as informações de falha nele contidas, desde que respeitadas as particularidades de cada método de demodulação. Em geral a frequência de amostragem pôde ser reduzida a $640 \mathrm{~Hz}$ para ambas as técnicas utilizadas.

A transformada de Hilbert foi mais robusta para a demodulação, permitindo que a análise fosse realizada com 
apenas 16384 amostras, sem perda de informação para quaisquer condições de carga do motor.

A redução do número de amostras do sinal trouxe uma notória diferença quanto ao número de operações necessários na análise de falhas, que resulta em um ganho computacional em termos de velocidade de processamento e armazenamento de dados.

\section{AGRADECIMENTOS}

Os autores agradecem ao Programa de pós-graduação em engenharia elétrica UFSJ/CEFET MG e a CAPES pelo apoio financeiro.

\section{REFERÊNCIAS}

Abd-el-Malek, M., Abdelsalam, A. K., \& Hassan, O. E. (2017). Induction motor broken rotor bar fault location detection through envelope analysis of start-up current using Hilbert transform. Mechanical Systems and Signal Processing, 93, 332-350.

Akin, B., Choi, S., Orguner, U., \& Toliyat, H. A. (2011). A simple real-time fault signature monitoring tool for motor-drive-embedded fault diagnosis systems. IEEE Transactions on Industrial Electronics, 58(5), 1990-2001.

Aydin, I., Karakose, M., \& Akin, E. (2011). A new method for early fault detection and diagnosis of broken rotor bars. Energy Conversion and Management, 52(4), 17901799 .

Batista, F. B., Lamim Filho, P. C. M., Pederiva, R., \& Silva, V. A. D. (2016). An empirical demodulation for electrical fault detection in induction motors. IEEE Transactions on Instrumentation and Measurement, 65(3), 559-569.

Bonnett, A. H., \& Yung, C. (2008). Increased efficiency versus increased reliability. IEEE Industry Applications Magazine, 14(1), 29-36.

Chakkor, S., Baghouri, M., \& Hajraoui, A. (2015). Enhanced ESPRIT Algorithm for Optimal Wind Turbine Fault Recognizing. Int. J. Comput. Sci. Theor. App, 3(1), 2939.

De Jesus Romero-Troncoso, R. (2017). Multirate signal processing to improve FFT-based analysis for detecting faults in induction motors. IEEE Transactions on industrial informatics, 13(3), 1291-1300.

Gao, Z., Cecati, C., \& Ding, S. X. (2015). A survey of fault diagnosis and fault-tolerant techniques-Part I: Fault diagnosis with model-based and signal-based approaches. IEEE Transactions on Industrial Electronics, 62(6), 3757-3767.

Gyftakis, K. N., \& Kappatou, J. C. (2014). The zero-sequence current as a generalized diagnostic mean in $\Delta$-connected three-phase induction motors. IEEE Transactions on Energy conversion, 29(1), 138-148.

Gyftakis, K. N., Antonino-Daviu, J. A., Garcia-Hernandez, R., McCulloch, M. D., Howey, D. A., \& Cardoso, A. J. M. (2016). Comparative experimental investigation of broken bar fault detectability in induction motors. IEEE Transactions on Industry Applications, 52(2), 14521459.

Lamim Filho, P. C. M., Batista, F. B., Pederiva, R., \& Silva, V. A. D. (2016). Electrical fault diagnosis in induction motors using local extremes analysis. Journal of Quality in Maintenance Engineering, 22(3), 321-332.

Lathi, B. P. (2006). Sinais e Sistemas Lineares-2. Bookman.

Puche-Panadero, R., Pineda-Sanchez, M., Riera-Guasp, M., Roger-Folch, J., Hurtado-Perez, E., \& Perez-Cruz, J. (2009). Improved resolution of the MCSA method via Hilbert transform, enabling the diagnosis of rotor asymmetries at very low slip. IEEE Transactions on Energy Conversion, 24(1), 52-59.

Puche-Panadero, R., Sarkimaki, V., \& Rodriguez, P. (2012, June). Detection of broken rotor bar fault in induction machine fed by frequency converter. In International Symposium on Power Electronics Power Electronics, Electrical Drives, Automation and Motion (pp. 10271032). IEEE.

Sapena-Bano, A., Pineda-Sanchez, M., Puche-Panadero, R., Perez-Cruz, J., Roger-Folch, J., Riera-Guasp, M., \& Martinez-Roman, J. (2015). Harmonic order tracking analysis: A novel method for fault diagnosis in induction machines. IEEE Transactions on Energy Conversion, 30(3), 833-841. 